International Journal of Wavelets, Multiresolution

and Information Processing

Vol. 16, No. 6 (2018) 1899001 (5 pages)

(C) World Scientific Publishing Company

DOI: $10.1142 / \mathrm{S} 0219691318990011$

\title{
Author index (Vol. 16)
}

Agajo, J., see Dibal

Akhtar, P., see Zia

Alenoghena, C. O., see Dibal

Alijani, A., Nonorthogonal fusion frames in Hilbert $C^{*}$ modules

Alim, A., Naseem, I., Togneri, R. and Bennamoun, M. The most discriminant subbands for face recognition: A novel informationtheoretic framework

An, J., see Gao

An, J., see Kai

Andrianov, P., On sufficient frame conditions for periodic wavelet systems

Angadi, L. M., see Shiralashetti

Aziz, A., see Zia

Banyarani, J. S., see Khosravi

Bennamoun, M., see Alim

Bhate, H., see Deshpande

Biuk-Aghai, R. P., see Luo

Cao, K. and Liu, Y., On the Reynaud-BouretRivoirard-Tuleau-Malot problem

Chang, Y., see Xie

Chen, D.-R., see Chen

Chen, F., see Xie

Chen, H., Huang, W. and Chen, D.-R., Simultaneous estimations of optimal directions and optimal transformations for functional data
16 (2018) 1850009 16 (2018) 1850048

16 (2018) 1850009

16 (2018) 1850015

16 (2018) 1850040 16 (2018) 1840006 16 (2018) 1840007

16 (2018) 1850002

16 (2018) 1850046

16 (2018) 1850048

16 (2018) 1850051

16 (2018) 1850040

16 (2018) 1850020

16 (2018) 1850016

16 (2018) 1850038 16 (2018) 1850034

16 (2018) 1850043

16 (2018) 1840013

16 (2018) 1850043
Chen, F., Zou, B. and Chen, N., The consistency of least-square regularized regression with negative association sequence

Chen, N., see Chen

Chen, X., see Liu

Chen, X., see $\mathrm{Xu}$

Chen, Y., Lai, Z., Wen, J. and Gao, C., Nuclear norm based two-dimensional sparse principal component analysis

Cheng, J., see Liu

Chiann, C., see Montoril

Cui, L., see $\mathrm{Xu}$

Deng, L., Shi, J. and Wang, Y., Robust video-based face recognition via $\mathrm{M}$ estimator and image set collaborative representation

Deshi, A. B., see Shiralashetti

Deshpande, R. and Bhate, H., Some crystallographic Haar type composite dilation wavelets for $P_{4}=$ $C_{4} \ltimes \mathbb{Z}^{2}$

Dibal, P. Y., Onwuka, E. N., Agajo, J. and Alenoghena, C. O., Enhanced discrete wavelet packet sub-band frequency edge detection using Hilbert transform

Du, Y., see Xie

Feise, F. and Sawatzki, L., Inhomogeneous shearlet coorbit spaces
16 (2018) 1850019

16 (2018) 1850019

16 (2018) 1850018

16 (2018) 1850039

16 (2018) 1840002

16 (2018) 1850018

16 (2018) 1850004

16 (2018) 1850039

16 (2018) 1840011

16 (2018) 1850046

16 (2018) 1850020

16 (2018) 1850009

16 (2018) 1850034

16 (2018) 1850026 
Gao, C., see Chen

Gao, W., Zhao, X., An, J. and Zou, J., Multipose $3 \mathrm{D}$ facial texture refinement for face recognition

Gao, W., see Mei

García, A. Hernández-Medina, M. A. and PérezVillalón, G., Filter banks on discrete abelian groups

Goyal, K., see Sharma

Guo, Y. and Li, B.-Z., The linear canonical wavelet transform on some function spaces

Hassan, N. M. H., see Nashat

Hernández-Medina, M. A., see García

Huang, L., see Sun

Huang, T., see Li

Huang, W., Liu, L., Yang, Z. and Zhao, Y., Sparse signal recovery via non-convex optimization and overcomplete dictionaries

Huang, W., see Chen

Huang, Y., see Zhang

Huo, L.-Z. and Tang, P., A graph-based active learning method for classification of remote sensing images

Hwan, R. U., see Il

Iglewska-Nowak, I., Uncertainty product of the spherical Gauss-Weierstrass wavelet

Il, K. K., Hwan, R. U. and Pil, C. B., An appropriate thresholding method of wavelet denoising for dropping ambient noise

Jakobsen, M. S., see Khedmati

Jian, M., see Wu

Jindal, H., Kasana, S. S. and Saxena, S., Underwater pipelines panoramic image transmission and refinement using acoustic sensors
16 (2018) 1840002

16 (2018) 1840006

16 (2018) 1850021

16 (2018) 1850029

16 (2018) 1850032

16 (2018) 1850010

16 (2018) 1850003

16 (2018) 1850029

16 (2018) 1840003

16 (2018) 1850053

16 (2018) 1850058

16 (2018) 1850043

16 (2018) 1850037

16 (2018) 1850023

16 (2018) 1850012

16 (2018) 1850030

16 (2018) 1850012

16 (2018) 1850017

16 (2018) 1840001

16 (2018) 1850013
Jyoti and Vashisht, L. K., On WH-packets of matrix-valued wave packet frames in $L^{2}\left(\mathbb{R}^{d}, \mathbb{C}^{s \times r}\right)$

16 (2018) 1850022

Kai, W., An, J., Zhao, X. and Zou, J., Accurate landmarking from 3D facial scans by CNN and cascade regression

16 (2018) 1840007

Kansal, I. and Kasana, S. S., Fusion-based image de-fogging using dual tree complex wavelet transform

Kasana, S. S., see Jindal

Kasana, S. S., see Kansal

Khedmati, Y. and Jakobsen, M. S., Approximately dual and perturbation results for generalized translationinvariant frames on LCA groups

Khosravi, A. and Banyarani, J. S., Some properties of $\mathrm{g}$-frames in Banach spaces

Kittisuwan, P., Lowcomplexity image denoising based on mixture model and simple form of MMSE estimation

Kovalyov, I. and Lebedeva, E., Uncertainty product for Vilenkin groups

Kundi, D. E. S., see Zia

Lai, Z., see Chen

Lebedeva, E., see Kovalyov

Leng, J., see $\mathrm{Li}$

Li, B.-Z., see Guo

Li, D., Leng, J. and Huang, T., New characterizations of gframes and g-Riesz bases

Li, L., see Yang

Li, R. and Zhou, J., Sparse time-frequency representation based on multiwindow discrete Gabor transform
16 (2018) 1850054

16 (2018) 1850013

16 (2018) 1850054

16 (2018) 1850017

16 (2018) 1850051

16 (2018) 1850052

16 (2018) 1850036

16 (2018) 1850048

16 (2018) 1840002

16 (2018) 1850036

16 (2018) 1850053 16 (2018) 1850010

16 (2018) 1850053

16 (2018) 1892001

16 (2018) 1850041 
Li, Y., see Zheng

Li, Y.-Z., see Zhao

Lin, B., see Liu

Lin, G., Yang, M., Shen, L., Yang, M. and Xie, M., Robust and discriminative dictionary learning for face recognition

Liu, C., Shang, Z., Lin, B. and Tang, Y. Y., A semantic tree method for image classification and video action recognition

Liu, C., see Sun

Liu, L., Yang, X., Wei, B. and $\mathrm{Wu}$, L., $\mathrm{A}$ new class of quaternary generalized cyclotomic sequences of order $2 d$ and length $2 p^{m}$ with high linear complexity

Liu, L., see Huang

Liu, P., see Wu

Liu, Q., see Sun

Liu, Y., Chen, X., Cheng, J., Peng, H. and Wang, Z., Infrared and visible image fusion with convolutional neural networks

Liu, Y., see Cao

Lou, Z., see Yang

Luo, B., see $\mathrm{Xu}$

Luo, H., Tang, Y Y., Biuk-Aghai, R. P., Yang, X., Yang, L. and Wang, Y., Wavelet-based extended morphological profile and deep autoencoder for hyperspectral image classification

Mei, S. and Gao, W., Shannon-Cosine wavelet spectral method for solving fractional FokkerPlanck equations

Montoril, M. H., Morettin, P. A. and Chiann, C., Wavelet estimation of functional coefficient regression models

Morettin, P. A., see Montoril
16 (2018) 1850014

16 (2018) 1850056

16 (2018) 1840008

16 (2018) 1840004

16 (2018) 1840008

16 (2018) 1840003

16 (2018) 1850006

16 (2018) 1850058

16 (2018) 1850008

16 (2018) 1840009

16 (2018) 1850018

16 (2018) 1850038

16 (2018) 1850044

16 (2018) 1850039

16 (2018) 1850016

16 (2018) 1850021

16 (2018) 1850004

16 (2018) 1850004
Naik, K. and Pal, A. K., A cryptosystem for lossless/lossy grayscale images in IWT domain using chaotic mapbased generated key matrices

16 (2018) 1850024

Naseem, I., see Alim

Nashat, A. A. and Hassan, N. M. H., Automatic segmentation and classification of olive fruits batches based on discrete wavelet transform and visual perceptual texture features

Nashat, A. A., Facial expression recognition using best tree RDLGP encoded features and HMM

Nisar, S. and Tariq, M., Dialect recognition for low resource language using an adaptive filter bank

Onwuka, E. N., see Dibal

Osgooei, E. and Rahimi, A., Gram matrix associated to controlled frames

Pal, A. K., see Naik

Pang, M. and Sun, H., Distributed learning with partial coefficients regularization

Pathak, A. and Singh, G. P., Wavelets in Sobolev space over local fields of positive characteristics

Peng, H., see Liu

Pérez-Villalón, G., see García

Pil, C. B., see Il

Prasad, A. and Verma, S. K., Continuous wavelet transform associated with zeroorder Mehler-Fock transform and its composition

Priyadarshi, G., see Rathish Kumar

Qi, W., see $\mathrm{Wu}$

Rahimi, A., see Osgooei

Rahimi, A., see RashidiKouchi
16 (2018) 1850040

16 (2018) 1850003

16 (2018) 1850047

16 (2018) 1850031

16 (2018) 1850009

16 (2018) 1850035

16 (2018) 1850024

16 (2018) 1850025

16 (2018) 1850027

16 (2018) 1850018

16 (2018) 1850029

16 (2018) 1850012

16 (2018) 1850050

16 (2018) 1850045

16 (2018) 1840001

16 (2018) 1850035

16 (2018) 1850057 
Raja, S. P., Secured medical image compression using DES encryption technique in Bandelet multiscale transform

Rashidi-Kouchi, M., Rahimi, A. and Shah, F. A., Duals and multipliers of controlled frames in Hilbert spaces

Rathish Kumar, B. V. and Priyadarshi, G., Wavelet Galerkin method for fourthorder multidimensional elliptic partial differential equations

Rekatsinas, N. and Stevenson, R., A quadratic finite element wavelet Riesz basis

Rinoshika, A., see Zheng

Saini, B. S., see Verma

Saini, I., see Verma

Sawatzki, L., see Feise

Saxena, S., see Jindal

Shah, F. A., see RashidiKouchi

Shang, Z., see Liu

Sharma, D. and Goyal, K., Second-generation wavelet optimized finite difference method (SGWOFD) for solution of Burger's equation with different boundary conditions

Shen, L., see Lin

Shi, J., see Deng

Shiralashetti, S. C., Angadi, L. M. and Deshi, A. B., Waveletbased lifting scheme for the numerical solution of some class of nonlinear partial differential equations

Singh, G. P., see Pathak

Smith, D. H., Wavelet spectra and filtering of tropical cyclone forecast errors

Stevenson, R., see Rekatsinas

Sun, H., see Pang
16 (2018) 1850028

16 (2018) 1850057

16 (2018) 1850045

16 (2018) 1850033

16 (2018) 1850055

16 (2018) 1850049

16 (2018) 1850049

16 (2018) 1850026

16 (2018) 1850013

16 (2018) 1850057

16 (2018) 1840008

16 (2018) 1850032

16 (2018) 1840004

16 (2018) 1840011

16 (2018) 1850046

16 (2018) 1850027

16 (2018) 1850011

16 (2018) 1850033

16 (2018) 1850025
Sun, H., see Wu

Sun, X., Huang, L. and Liu, C., Multispectral face spoofing detection using VIS-NIR imaging correlation

Sun, Y. and Liu, Q., Attribute recognition from clothing using a Faster R-CNN based multitask network

Suo, J., see Zheng

Tang, P., see Huo

Tang, Y. Y., see Liu

Tang, Y. Y., see Luo

Tang, Y. Y., see Zheng

Tang, Y., see Xie

Tang, Y., see Xu

Tariq, M., see Nisar

Togneri, R., see Alim

Vashisht, L. K., see Jyoti

Verma, A. K., Saini, I. and Saini, B. S., The baseline wandering noise removal from ECG signal using forwardbackward Riemann Liouville fractional integral-based empirical wavelet transform approach

Verma, S. K., see Prasad

Wang, B., see Zhou

Wang, J., Mathematical analysis on out-ofsample extensions

Wang, Y. and Zheng, $\mathrm{X}$., Cross-device hand vein recognition based on improved SIFT

Wang, Y., see Deng

Wang, Y., see Luo

Wang, Z., see Liu

Wei, B., see Liu

Wen, J., see Chen

Wu, L., Xu, Y., Jian, M., Xu, X. and Qi, W., Face liveness detection scheme with static and dynamic features

Wu, L., see Liu

Wu, Y., Sun, H. and Liu, P., A novel fast detection method of infrared LSS-Target in complex urban background
16 (2018) 1850008

16 (2018) 1840003

16 (2018) 1840009

16 (2018) 1850055

16 (2018) 1850023

16 (2018) 1840008

16 (2018) 1850016

16 (2018) 1850014

16 (2018) 1850034

16 (2018) 1850039

16 (2018) 1850031

16 (2018) 1850040

16 (2018) 1850022

16 (2018) 1850049

16 (2018) 1850050

16 (2018) 1850007

16 (2018) 1850042

16 (2018) 1840010

16 (2018) 1840011

16 (2018) 1850016

16 (2018) 1850018

16 (2018) 1850006

16 (2018) 1840002

16 (2018) 1840001

16 (2018) 1850006

16 (2018) 1850008 
Xiao, J., see $\mathrm{Xu}$

Xie, H., Du, Y., Yu, H., Chang, Y., Xu, Z. and Tang, Y., Open set face recognition with deep transfer learning and extreme value statistics

Xie, M., see Lin

Xie, T. and Chen, F. Non-convex clustering via proximal alternating linearized minimization method

Xu, L., Tang, Y., Luo, B., Cui, L., Chen, $\mathrm{X}$. and Xiao, J., A combined WeisfeilerLehman graph kernel for structured data

$\mathrm{Xu}, \mathrm{T}$. and Zhou, Y., Elders' fall detection based on biomechanical features using depth camera

$\mathrm{Xu}, \mathrm{X}$., see $\mathrm{Wu}$

$\mathrm{Xu}, \mathrm{Y}$., see $\mathrm{Wu}$

$\mathrm{Xu}, \mathrm{Z}$., see $\mathrm{Xie}$

Yang, L., see Luo

Yang, M., see Lin

Yang, Q. and Lou, Z., Commutators and rough kernels without zero homogeneous condition

Yang, S., see Zheng

Yang, X., see Liu

Yang, X., see Luo

Yang, Z. and Li, L., Approximation by Riesz Means on the Rotation Group $S O(3)$

Yang, Z., see Huang

Yin, X.-C., see Zhu

Younus Bhat, M., Necessary condition and sufficient conditions for nonuniform wavelet frames in $L^{2}(K)$

$\mathrm{Yu}, \mathrm{H}$., see Xie

Zakharov, V. G., Rotation properties of
16 (2018) 1850039

16 (2018) 1850034

16 (2018) 1840004

16 (2018) 1840013

16 (2018) 1850039

16 (2018) 1840005

16 (2018) 1840001

16 (2018) 1840001

16 (2018) 1850034

16 (2018) 1850016

16 (2018) 1840004

16 (2018) 1850044

16 (2018) 1850014

16 (2018) 1850006

16 (2018) 1850016

16 (2018) 1892001

16 (2018) 1850058

16 (2018) 1840012

16 (2018) 1850005

16 (2018) 1850034
$2 \mathrm{D}$ isotropic dilation matrices

Zhang, K., Huang, Y. and Zhao, C., Remote sensing image fusion via RPCA and adaptive $\mathrm{PCNN}$ in NSST domain

Zhao, C., see Zhang

Zhao, J. and Li, Y.-Z., A class of vector-valued subspace weak Gabor duals of type II

Zhao, X., see Gao

Zhao, X., see Kai

Zhao, Y., see Huang

Zheng, X., Yang, S., Tang, Y. Y. and Li, Y., Parseval transforms for finite frames

Zheng, X., see Wang

Zheng, Y., Rinoshika, A. and Suo, J., Waveletbased phase average on the multi-scale wake structures of square cylinder

Zhou, J., see Li

Zhou, X. and Wang, B., Wavelet analysis on some smooth surface with nonzero constant Gaussian curvature

Zhou, Y., see Xu

Zhu, C. and Yin, X.-C., Effective human detection via multimodel classification and adaptive late fusion

Zia, R., Akhtar, P., Aziz, A., Kundi, D. E. S., Multiresolution transformsbased hybrid feature extraction technique for differentiating glioma grades

Zou, B., see Chen

Zou, J., see Gao

Zou, J., see Kai
16 (2018) 1850001

16 (2018) 1850037

16 (2018) 1850037

16 (2018) 1850056

16 (2018) 1840006

16 (2018) 1840007

16 (2018) 1850058

16 (2018) 1850014

16 (2018) 1840010

16 (2018) 1850055

16 (2018) 1850041

16 (2018) 1850007

16 (2018) 1840005

16 (2018) 1840012

16 (2018) 1850048

16 (2018) 1850019

16 (2018) 1840006

16 (2018) 1840007 